\title{
Analgesic Effect of Perioperative Duloxetine in Patients After Total Knee Arthroplasty: A Prospective, Randomized, Double-Blind, Placebo- Controlled Trial
}

\author{
Mingcheng Yuan \\ West China Hospital/West China School of Medicine, Sichuan University \\ Tingting Tang \\ West China Hospital/West China School of Medicine, Sichuan University \\ Zichuan Ding \\ West China Hospital/West China School of Medicine, Sichuan University \\ Hao Li \\ West China Hospital/West China School of Medicine, Sichuan University \\ Zongke Zhou ( zongkehx@126.com ) \\ West China Hospital/West China School of Medicine, Sichuan University
}

\section{Research Article}

Keywords: total knee arthroplasty, duloxetine, analgesic effect, randomized controlled trial

Posted Date: November 29th, 2021

DOI: https://doi.org/10.21203/rs.3.rs-1020353/v1

License: (c) (i) This work is licensed under a Creative Commons Attribution 4.0 International License.

Read Full License

Version of Record: A version of this preprint was published at BMC Musculoskeletal Disorders on March 12th, 2022. See the published version at https://doi.org/10.1186/s12891-022-05194-z. 


\section{Abstract}

Background: To investigate the analgesic effect of perioperative use of duloxetine in patients received total knee arthroplasty (TKA).

Method: The hospital pharmacy prepared small capsules containing either duloxetine or starch (placebo) which were all identical in appearance and weight (1:1). Enrolled patients were given a capsule (containing either $60 \mathrm{mg}$ duloxetine or $60 \mathrm{mg}$ placebo) every night before sleep since preoperative day 2 till postoperative day 14 (17 days in all) by a nurse who were not involved in this trial. Other perioperative managements were the same in the two groups. The primary outcome was the VAS score (both rVAS and aVAS) throughout the perioperative period. The secondary outcomes included opioid consumption, range of motion, including both active ROM (aROM) and passive ROM (pROM) and adverse events.

Result: rVAS in duloxetine group were significantly less than placebo group throughout the postoperative period (From postoperative 2 hours to postoperative 3 months) $(P<0.05)$. In terms of aVAS, similarly, duloxetine group had less aVAS than placebo group throughout the postoperative period (From postoperative 6 hours to postoperative 3 months) $(P<0.05)$. During the postoperative period (From postoperative day 1 to 7 ), patients in duloxetine group consumed significantly less opioids per day than the placebo group $(P<0.05)$. aROM in duloxetine group were significantly better than placebo group from postoperative 6 hours to postoperative day $5(P<0.05)$, since postoperative day 6 , the aROM became comparable between the two groups $(P>0.05)$. In terms of $p R O M$, duloxetine group had significantly better pROM from postoperative 6 hours to postoperative day $4(P<0.05)$, thereafter, the pROM between the two groups became comparable $(P>0.05)$. No significant difference was found between the two groups in the rates of dizziness, bleeding, sweating, fatigue and dryness of mouth. In the placebo group, more patients got nausea/vomiting and constipation $(P<0.05)$. However, in terms of drowsiness, duloxetine group was reported higher rate $(P<0.05)$.

Conclusion: Duloxetine could reduce acute postoperative pain and decrease the opioids consumption as well as accelerating postoperative recovery, without increasing the risk of adverse medication effects in patients undergoing TKA. Duloxetine could act as a good supplement in multimodal pain management protocol for patients undergoing TKA.

Trial registration statement: This study was registered in the Chinese Clinical Trial Registry (ChiCTR2000033910). The date of registration was 06/16/2020.

\section{Background}

Total knee arthroplasty (TKA) is a mature surgery relieving pain as well as improving the function and the life quality of patients, but the pain following TKA is still severe and persistent(1). Inadequate pain control would delay rehabilitation and increase the risk of postoperative complications(2). It is also reported that improper and inadequate postoperative pain management is associated with longer hospital stays, increased rates of unanticipated hospital admissions and readmissions due to pain and an overall 
increase cost of care(3). Despite the multimodal analgesia, mostly including acetaminophen, pregabalin, and nonsteroidal anti-inflammatory drugs (NSAIDs), is commonly applied in TKA patients, it is reported that over $20 \%$ of patients undergoing TKA are still dissatisfied, most of which are for the pain(4). Furthermore, despite recent advances in multimodal analgesia that reduce opioid consumption, the general use of opioid is still prevalent. Therefore, the current multimodal pain management following TKA still needs optimizing.

Duloxetine (Cymbalta), a potent selective serotonin and norepinephrine dual reuptake inhibitor (SNRI), has been widely approved for major depressive disorder, generalized anxiety disorder, diabetic peripheral neuropathy, fibromyalgia, and chronic musculoskeletal pain(5). It can increase the levels of both serotonin and norepinephrine by inhibiting the reuptake and finally enhanced the descending inhibitory pain pathways in the central nervous system $(6,7)$. Duloxetine's analgesia effect is independent of its antidepression effect, it has similar analgesia effects on both depressed and nondepressed patients. Many prior studies have already demonstrated the analgesic effect of duloxetine in patients experiencing chronic musculoskeletal pain such as osteoarthritis knee pain(8), but the peri-TKA use of duloxetine for postoperative pain relief remains controversial: Some studies reported that patients with perioperative use of duloxetine experienced better pain relief after TKA during postoperative weeks 2 to 12, but no apparent benefit was observed in terms of reduced pain or opioid consumption during the immediate postoperative period (weeks 1 and 2) (9), however, some others found that the post-TKA pain of patients with ambulation, flexion, and at rest did not differ between groups throughout postoperative weeks 1 to 6 , while the perioperative use of duloxetine could reduce the postoperative daily opioid consumption(10).

Therefore, we performed a prospective randomized, double-blind, placebo-controlled trial in patients undergoing unilateral primary TKA to investigate the analgesic effect of perioperative use of duloxetine. We hypothesized that duloxetine would reduce postoperative pain and morphine consumption relative to placebo, the primary outcome being postoperative VAS scores, including VAS at rest (rVAS) and VAS upon ambulation (aVAS).

\section{Patients And Methods}

This study was registered in the Chinese Clinical Trial Registry (ChiCTR2000033910). The date of registration was 06/16/2020. Approval was obtained from the Clinical Trials and Biomedical Ethics Committee of West China Hospital, and written informed consent was obtained from all patients before participation in this prospective randomized, double-blind, placebo-controlled trial.

\section{Patients}

Patients who were diagnosed with knee osteoarthritis and scheduled to undergo primary unilateral TKA were eligible for study enrollment. Patients were excluded according to the following criteria: American Society of Anesthesiologists physical status IV, a known psychiatric disorder, Hamilton Depression Scale (HAMD) and Hamilton Anxiety Scale (HAMA) scores both $<7$, intolerance or allergy to any of the study 
drugs, alcohol or opioid dependence. ongoing anticoagulant treatment, preoperative hepatic or renal dysfunction, serious cardiac and/or cerebrovascular comorbidities and refusal to participate. Patients were additionally excluded for concurrent use of duloxetine or other SNRIs, monoamine oxidase inhibitors, tricyclic antidepressants, triptans, lithium, or buspirone.

\section{Randomization and Blinding}

Group assignment was concealed from the patients, the nurses, the treating surgeons, the data collectors and the statistician. The hospital pharmacy was provided a random allocation sequence which was computer-generated and concealed in consecutively numbered, opaque, sealed envelopes by a statistician not involved in the data analysis. An experienced surgeon enrolled the patients, and another surgeon reviewed the inclusion criteria and recorded basic information. The hospital pharmacy prepared small capsules containing either duloxetine or starch (placebo) which were all identical in appearance and weight (1:1). All the capsules were given to patients every night before sleep by a nurse who were not involved in this trial.

\section{Surgical Procedure and Perioperative Management}

All the TKAs were performed by the same surgical team. A midline skin incision, a standard medial parapatellar approach and a cemented total knee system-Attune (DePuy, IN, USA) were used in all patients. No tourniquet or drain were applied. Ten milligrams $/ \mathrm{kg}$ of tranexamic acid (TXA) was given 10 min before skin incision by intravenous infusion, followed by $10 \mathrm{mg} / \mathrm{kg}$ administered by local injection when closing the incision; Two doses of $10 \mathrm{mg} / \mathrm{kg}$ of TXA were separately administered 3 and 6 hours after surgery to reduce perioperative blood loss(11). Thromboprophylaxis was started in a timely manner: a half dose of low-molecular weight heparin (LMWH, $0.2 \mathrm{~mL} 2,000 \mathrm{IU})$ was given to patients $6 \mathrm{~h}$ postoperatively, and a full dose $(0.4 \mathrm{~mL} 4,000 \mathrm{IU})$ was given at $24 \mathrm{~h}$ intervals in the subsequent days by subcutaneous injection till the day of discharge(12). The criterion of blood transfusion was set as an Hemoglobin level of $<70$ or $70 \sim 100 \mathrm{~g} / \mathrm{L}$ but with symptomatic anemia (defined as severe mental status changes, palpitations, and/or pallor).

\section{Analgesic Protocol and intervention drug}

Patients received standardized general anesthesia and basic analgesic protocol. From preoperative day 2 to the day before surgery, patients were given celecoxib $200 \mathrm{mg}$ twice a day (one dose after breakfast, the other one after dinner) for preemptive analgesia(13). Intraoperatively, all patients received general anesthesia which was induced by sufentanil $0.5 \mu \mathrm{g} / \mathrm{kg}$, midazolam $0.04 \mathrm{mg} / \mathrm{kg}$, propofol $1-2 \mathrm{mg} / \mathrm{kg}$, and Cisatracurium $2 \mu \mathrm{g} / \mathrm{kg}$ intravenously, followed by continuous intravenous infusion of remifentanil $0.1-$ $0.3 \mu \mathrm{g} /(\mathrm{kg} \cdot \mathrm{min})$, propofol $2-5 \mathrm{mg} /(\mathrm{kg} \cdot \mathrm{h})$ and inhalation of sevoflurane to maintain anesthesia. Additionally, an $80-\mathrm{mL}$ periarticular injection of $0.25 \%$ ropivacaine was administered to all patients for local infiltration analgesia. Since postoperative day 1 , the protocol of oral celecoxib restarted till postoperative 3 weeks when the patients came back to hospital for taking out the stitches. If acute or resistant pain occurred (VAS>6), the opioids (oral oxycodone or subcutaneous morphine) were used as rescue analgesics. 
Patients were given a capsule (containing either $60 \mathrm{mg}$ duloxetine or $60 \mathrm{mg}$ placebo) every night before sleep since preoperative day 2 till postoperative day 14 (17 days in all). The dose of duloxetine, $60 \mathrm{mg}$, was determined according to the Cochrane database review of duloxetine for painful neuropathy or chronic pain which indicated that the proper dose of oral duloxetine was $60 \mathrm{mg}$ daily, for $20 \mathrm{mg}$ was ineffective and $120 \mathrm{mg}$ was no more effective than $60 \mathrm{mg}$ with more adverse events(5).

\section{Outcome measurements}

The primary outcome was the VAS score (both rVAS and aVAS) throughout the perioperative period. The secondary outcomes included opioid consumption, range of motion, including both active ROM (aROM) and passive ROM (pROM) and adverse events.

The VAS score was collected preoperatively and at 2, 4, 6, 24, 36, and $48 \mathrm{~h}$ after surgery by a project nurse (2, 4, $6 \mathrm{~h}$ after surgery without aVAS), thereafter the average of the VAS in the morning (when getting up) and evening (before going to bed) from postoperative days 2 to 7,3 weeks and 3 months were also collected. The opioid consumption was recorded every day from the day of surgery to postoperative day 7 by a project nurse, all the opioids no matter oral oxycodone or subcutaneous morphine were converted into morphine equivalent. The ROM was recorded at $6,24,36,48 \mathrm{~h}$ after surgery and then from postoperative days 2 to 7, 3 weeks and 3 months in the evening. Finally, adverse events, defined on the basis of previous studies regarding the safety of duloxetine, were recorded since the trial started.

\section{Statistical Analysis}

Distributions of demographic data, baseline data, and primary and secondary outcomes were assessed using measures of central tendency (mean, standard deviation) for quantitative variables and with percentages for qualitative variables. The student $t$ test or the Wilcoxon signed-rank test was used to analyze continuous variables, and the chi-square test or the Fisher exact test was used to determine differences in categorical variables. All data analyses were performed using SPSS (version 23.0; IBM). Significance was set at $\mathrm{P}<0.05$.

\section{Sample-Size Calculations}

The sample-size estimate was based on the primary outcome (VAS pain score). To determine the necessary sample size for sufficient statistical power, we used the results of the previous study of our center(14) which showed that 5.2 \pm 2.5 was the mean post-TKA VAS score. A 2-point difference was considered to be clinically relevant because approximately 2 points is the mean acceptable VAS pain score postoperatively(15). Assuming a common within-group SD of 2.5 points, we calculated that a sample size of 42 patients per group would provide $95 \%$ power at a 2-sided a of 0.05 to detect a 2-point difference in mean VAS pain score between the duloxetine and placebo groups using a two-sample t test. Furthermore, the sample size was increased by $20 \%$ to compensate for expected dropouts, resulting in 50 patients per group and a total number of 100 patients.

\section{Result}


From June 8th 2020 to July 31 st 2020, 131 patients were scheduled to undergo a total knee arthroplasty at our institution. Among these patients, 19 did not meet the inclusion criteria, 12 declined to participate. Hence, the remaining 100 patients were randomized to two groups (50 patients in each group) (Fig. 1). No patient was lost or excluded during the follow-up period. No significant differences were identified among the groups in patient demographic and preoperative characteristics (Table 1).

Table 1

Demographic and preoperative data

\begin{tabular}{|llll|}
\hline & Duloxetine (50) & Placebo (50) & P \\
\hline Age & $67.8 \pm 10.12$ & $66.2 \pm 9.83$ & 0.430 \\
\hline Female & $30(60 \%)$ & $27(54 \%)$ & 0.622 \\
\hline Height & $1.58 \pm 0.08$ & $1.60 \pm 0.09$ & 0.243 \\
\hline Weight & $62.97 \pm 10.21$ & $63.13 \pm 9.92$ & 0.937 \\
\hline BMI & $24.67 \pm 4.35$ & $24.83 \pm 3.87$ & 0.846 \\
\hline rVAS & $3.47 \pm 1.77$ & $3.62 \pm 2.02$ & 0.694 \\
\hline aVAS & $6.31 \pm 2.35$ & $6.52 \pm 2.60$ & 0.673 \\
\hline aROM & $100.37 \pm 14.21$ & $102.72 \pm 13.52$ & 0.399 \\
\hline pROM & $104.12 \pm 14.69$ & $107.29 \pm 14.37$ & 0.278 \\
\hline ASA II/III & $43 / 7$ & $47 / 3$ & 0.182 \\
\hline HAMD & $3.2 \pm 1.1$ & $3.1 \pm 1.1$ & 0.650 \\
\hline HAMA & $3.1 \pm 1.3$ & $3.3 \pm 1.2$ & 0.426 \\
\hline Preoperative medications & & & \\
\hline NSAIDs & $12(24)$ & $15(30)$ & 0.499 \\
\hline Acetaminophen & $2(4)$ & $2(4)$ & 1.000 \\
\hline Opioids & 0 & 0 & 1.000 \\
\hline Antidepressant & 0 & 0 & 1.000 \\
\hline
\end{tabular}


Table 2

Adverse event

\begin{tabular}{|llll|}
\hline & Duloxetine $(\mathbf{n}=50)$ & Placebo $(\mathbf{n}=50)$ & P value \\
\hline Nausea/vomiting & $5(10)$ & $15(30)$ & 0.012 \\
\hline Dizziness & $3(6)$ & $5(10)$ & 0.461 \\
\hline Drowsiness & $20(40)$ & $10(20)$ & 0.029 \\
\hline Bleeding & $0(0)$ & $0(0)$ & N.A \\
\hline Sweating & $2(4)$ & $2(4)$ & 1.000 \\
\hline Fatigue & $23(46)$ & $26(52)$ & 0.548 \\
\hline Dryness of mouth & $18(36)$ & $17(34)$ & 0.834 \\
\hline Constipation & $3(6)$ & $11(22)$ & 0.021 \\
\hline
\end{tabular}

\section{Primary Outcome}

rVAS in duloxetine group were significantly less than placebo group throughout the postoperative period (From postoperative 2 hours to postoperative 3 months) ( $P<0.05$ ) (Fig. 2). In terms of aVAS, similarly, duloxetine group had less aVAS than placebo group throughout the postoperative period (From postoperative 6 hours to postoperative 3 months) $(P<0.05)$ (Fig. 3$)$.

\section{Secondary Outcomes}

During the postoperative period (From postoperative day 1 to 7 ), patients in duloxetine group consumed significantly less opioids per day than the placebo group $(P<0.05)$ (Fig. 4). aROM in duloxetine group were significantly better than placebo group from postoperative 6 hours to postoperative day $5(P<0.05)$, since postoperative day 6 , the aROM became comparable between the two groups ( $P>0.05)$ (Fig. 5). In terms of pROM, duloxetine group had significantly better pROM from postoperative 6 hours to postoperative day $4(P<0.05)$, thereafter, the pROM between the two groups became comparable $(P>0.05)$ (Fig. 6). Regarding adverse events, no significant difference was found between the two groups in the rates of dizziness, bleeding, sweating, fatigue and dryness of mouth. In the placebo group, more patients got nausea/vomiting and constipation $(P<0.05)$. However, in terms of drowsiness, duloxetine group was reported higher rate $(P<0.05)$ (Table. 2$)$.

\section{Discussion}

Nowadays, although multimodal analgesia strategies have been widely implemented to control postoperative pain in TKA patients, it was reported that over $20 \%$ of patients still suffered from severe postoperative pain(16). To deal with this annoying problem, surgeons never stopped to explore new methods. Recently, some researchers started to turn their eyes out of regular analgesia drugs, onto the 
application of antidepressant drugs like tricyclic antidepressants, selective serotonin reuptake inhibitors, and SNRIs, especially duloxetine. The latest review in 2014 which discussed the application of antidepressant drugs for postoperative pain concluded that there was insufficient evidence to support clinical use and called for more high-quality trials to confirm the exact effect of antidepressant drugs on postoperative pain(17). Duloxetine (Cymbalta), a potent selective SNRI, primarily found to treat depression, has already been demonstrated the analgesic effect in patients with chronic musculoskeletal pain(8). However, its effect on postoperative pain relief after TKA remains controversial.

So, we conducted this study to detect if duloxetine could be used as an adjuvants or supplement of multimodal analgesia strategies after TKA. In this trial of oral duloxetine $60 \mathrm{mg}$ administered daily for 17 days initiated on preoperative day 2 and continued for 14 days postoperatively, postoperative pain, including both rVAS and aVAS, was significantly reduced relative to placebo through the whole postoperative 3 months. Besides, the placebo group consumed markedly more morphine equivalent on during the postoperative period. In terms of secondary outcomes, postoperative recovery of function of the knee (range of motion at different time points) was better in the duloxetine group on the early days after surgery. When it comes to the side effects, patients took duloxetine had better sleep quality and less cases of nausea, vomit or constipation than placebo group, and the difference was marked.

SNRIs, like duloxetine, are targeted presynaptically and postsynaptically on serotonergic and noradrenergic nerve terminals and are the key regulator of synaptic serotonin and noradrenaline levels in central nervous system(18). The basic function of SNRIs is to block and then down-regulate serotonin and noradrenaline transporters, which therefore increases the concentrations of these two neurotransmitters(19). The increase of unbound serotonin and noradrenaline would enhance the descending inhibitory pain pathways in the central nervous system(6, 7). Although the antidepression effect of SNRIs is relatively independent with its analgesia effect, there are still some studies demonstrated that serotonergic signaling in brain regions relevant to affective cognition have been demonstrated to be coupled to tonic pain ratings in healthy volunteers $(20,21)$. It was reported that 7-14 days were necessary for SSRIs to take effect in treating anxiety or depression because the brain need time to establish the response(22, 23), so we hypothesized that it might likewise take days for SNRIs to take effect in relieving pain.

Lunn et al(1) randomized 120 patients scheduled for TKA in a double-blind manner to either $10 \mathrm{mg}$ escitalopram (a kind of SSRI) or placebo daily from pre-anesthesia to postoperative day 6 in addition to a standardized analgesic regime and found that no between-group differences were observed in overall pain upon ambulation from 4 to $48 \mathrm{~h}$ or overall pain at rest from 2 to $48 \mathrm{~h}$ after surgery. But conversely, overall pain upon ambulation and overall pain at rest from day 2 to 6 after surgery were lower in the escitalopram versus placebo group, which suggested that SSRI could relief postoperative pain but with a delayed attribute. Similarly, Koh et al(9) randomized 80 patients scheduled for TKA to either duloxetine group (30 mg from the day before surgery and for 6 weeks after surgery) or control group and finally found that patients received duloxetine experienced better pain control during postoperative weeks 2 to 12 , while there was no apparent benefit in terms of reduced pain during the immediate postoperative 
period (weeks 1 and 2). Koh et al attributed the delayed analgesia to the study design and to the already potent analgesic efficacy of their current multimodal regimen. According to the abovementioned trials, we hypothesized that it might take days as buffer time for duloxetine to take the effect of analgesia. So, we conducted this trial, initiating the intervention of duloxetine much earlier than previous studies $(60 \mathrm{mg}$ daily for 17 days initiated on preoperative day 2 and continued for 14 days postoperatively), so that the duloxetine might take effect quickly after surgery, when the pain was supposed to be severe. Consequently, we found that since postoperative $6 \mathrm{~h}$, both the rVAS and aVAS were significantly lower in duloxetine group till postoperative 3 months. It demonstrated that initiating duloxetine early preoperatively could effectively relief the pain of patients after TKA, which was consistent with prior studies and our initial hypothesis.

The opioids consumption, a parameter that measures the postoperative fulminant pain of patients, is entirely different from VAS, which commonly used to measure regular pain. We gave patients opioids only when the immediate VAS score exceeded 6 , and the opioids acted as a remedial treatment. As early as the 1980s, microinjection of 5-HT into the rostral ventromedial medulla (RVM) had already been demonstrated an antinociceptive function(24). During the same period, Mohrland et al(25) discovered that microinjection of a 5-HT neurotoxin into the RVM reduced the antinociceptive action of morphine. Similarly, norepinephrine also has well established antinociceptive effects when applied intrathecally(26), and Pang et al(27) found that the depletion of spinal norepinephrine by intrathecal 6-OH dopamine, which is a scavenger of NE, markedly attenuated the antinociceptive effect of morphine. According to the abovementioned studies, we hypothesized that the duloxetine, which is the reuptake inhibitor of both serotonin and norepinephrine could enhance the antinociceptive action of morphine or other opioids by increasing the concentration of unbound 5-HT and NE. In 2010, Ho et al(28) first conducted a randomized placebo-controlled trial in 50 patients who received either two doses of oral duloxetine $60 \mathrm{mg}(2 \mathrm{~h}$ before TKA and on the first day postoperatively) or placebo to specially detect the effect of duloxetine in morphine requirements after TKA and found that the morphine consumption during the $24 \mathrm{~h}$ and $48 \mathrm{~h}$ after TKA were significantly lower in the duloxetine group, compared with the placebo group. However, the sample size was small in Ho's study, and only two doses of duloxetine were administrated which could not totally reflect the effect of duloxetine during the postoperative period. Later in 2016, based on the study of $\mathrm{Ho}$ et al, YaDeau et al(10) implemented a longer duration of therapy starting from the day of surgery to the postoperative 14 days ( 1 dose of $60 \mathrm{mg}$ oral duloxetine daily, 15 doses in all) and found the opioids-reduced effect of duloxetine started quickly after administration. The total daily opioid consumption was significantly less in the duloxetine group from postoperative day 1 to 14 , compared with the placebo group, which was consistent with Ho's study. Similarly, in our study, we prolonged the application of duloxetine before surgery and found that the daily morphine equivalent was markedly less in the duloxetine group from postoperative day 1 to 7 , through the early postoperative period. To sum up, the duloxetine could effectively relief the fulminant pain after TKA and this function took effect quickly after application.

Range of motion, which acted as a measurement of recovery of knee function, was significantly better in the duloxetine group during the early postoperative period. Because, both VAS (rVAS and aVAS) and 
opioid consumption were significantly less in the duloxetine group, and the relatively milder pain would encourage patients in the duloxetine group to be more willing to exercise more frequently and hard, which would therefore accelerate the recovery of the knee function after surgery.

Tolerability and side effects are important issues in analgesic trials. It was reported by Lunn MP et al(5) that minor adverse effects are common with duloxetine and the regimen of duloxetine $60 \mathrm{mg}$ daily has lower rate of side effects than the dose of $120 \mathrm{mg}$ daily. In our study, although some mild side effects occurred, all the patients were tolerated to the duloxetine regimen of $60 \mathrm{mg}$ per night. Some patients in duloxetine group said they had more drowsiness at night. It is an interesting finding because the drowsiness at night isn't strictly a side effect, it was reported that a deep sleep at night could relief the nervous mind and unnecessary anxiety. Besides, it was reported by Blagestad et al(29) that the pain after surgery may obviously contribute to impaired sleep. Oppositely, an improved sleep has also been demonstrated antinociceptive effects(30). Of note, we noticed that the rates of nausea and vomit as well as constipation were markedly lower in the duloxetine group than in the placebo group. We inferred that the minimum in opioid use by duloxetine might therefore reduce the opioid-related adverse effects especially nausea, vomit and constipation. Although no serious side effects were observed, other studies have suggested an increased risk of perioperative bleeding from the cut and other postoperative morbidities(1). However, in our study, no such events were observed.

This study had several limitations, the most obvious being its confinement to Chinese patients, and most of them were women. The reason for this predominance of female patients in the Chinese TKA population is unknown. Second, the sample size of this study was calculated according to the primary outcome, so, it might be underpowered to detect other outcomes like rates of various side effects, which calls for future studies to investigate. Third, although we initiated the duloxetine intervention earlier than prior studies $(10,28)$, it still couldn't be determined whether the duloxetine exerted an effect exactly after surgery, where pain is more pronounced. So, future studies could focus on the optimal duration of perioperative use of duloxetine.

\section{Conclusion}

In conclusion, duloxetine could reduce acute postoperative pain and decrease the opioids consumption as well as accelerating postoperative recovery, without increasing the risk of adverse medication effects in patients undergoing TKA. Duloxetine could act as a good supplement in multimodal pain management protocol for patients undergoing TKA.

\section{Abbreviations}

TKA: total knee arthroplasty; BMI: body mass index; ASA: American Society of Anesthesiologists; rVAS: Visual Analogue Scale at rest; aVAS: Visual Analogue Scale upon ambulation; aROM: active range of motion; pROM: passive range of motion; HAMD: Hamilton Depression Scale; HAMA: Hamilton Anxiety Scale; TXA: tranexamic acid; LMWH: low-molecular weight heparin; RVM: rostral ventromedial medulla 


\section{Declarations}

\section{Ethics approval and consent to participate}

This trial was registered in the Chinese Clinical Trial Registry (ChiCTR2000033910). The date of registration was 06/16/2020. Approval was obtained from the Clinical Trials and Biomedical Ethics Committee of West China Hospital. The study was conducted at the Department of Joint Surgery of West China Hospital, Sichuan University, in accordance with the CONSORT (Consolidated Standards of Reporting Trials) Statement. Informed consent was obtained from all participants or, if participants are under 16 , from a parent and/or legal guardian.

all methods were carried out in accordance with relevant guidelines and regulations (Declaration of Helsinki)

\section{Consent for publication}

Not Applicable

\section{Availability of data and material}

Data used and analyzed in this study are available from the corresponding author on reasonable request.

\section{Competing interests}

The authors declare that they have no competing interests.

\section{Funding}

This work was supported by 1.3.5 project for disciplines of excellence, West China Hospital, Sichuan University (ZYJC18039) and Regional Innovation \& Cooperation program of Science \& Technology Department of Sichuan Province (No. 2021YFQ0028).

\section{Authors' contributions}

This study was conducted under the guidance of ZK-Z. The article was written by MC-Y and TT-T. The clinical assessment was done by ZC-D. The statistical analysis was performed by H-L. All authors read and approved the final manuscript.

\section{Acknowledgements}

Not Applicable

\section{References}


1. Lunn TH, Frokjaer VG, Hansen TB, Kristensen PW, Lind T, Kehlet H. Analgesic effect of perioperative escitalopram in high pain catastrophizing patients after total knee arthroplasty: a randomized, double-blind, placebo-controlled trial. Anesthesiology. 2015;122(4):884-94.

2. Andersen L, Gaarn-Larsen L, Kristensen BB, Husted H, Otte KS, Kehlet H. Subacute pain and function after fast-track hip and knee arthroplasty. Anaesthesia. 2009;64(5):508-13.

3. Parvizi J, Miller AG, Gandhi K. Multimodal pain management after total joint arthroplasty. J Bone Joint Surg Am. 2011;93(11):1075-84.

4. Carli F, Charlebois P, Stein B, Feldman L, Zavorsky G, Kim DJ, et al. Randomized clinical trial of prehabilitation in colorectal surgery. Br J Surg. 2010;97(8):1187-97.

5. Lunn MP, Hughes RA, Wiffen PJ. Duloxetine for treating painful neuropathy, chronic pain or fibromyalgia. Cochrane Database Syst Rev. 2014(1):Cd007115.

6. Fields HL, Heinricher MM, Mason P. Neurotransmitters in nociceptive modulatory circuits. Annu Rev Neurosci. 1991;14:219-45.

7. Woolf CJ. Pain: moving from symptom control toward mechanism-specific pharmacologic management. Ann Intern Med. 2004;140(6):441-51.

8. Chappell AS, Ossanna MJ, Liu-Seifert H, lyengar S, Skljarevski V, Li LC, et al. Duloxetine, a centrally acting analgesic, in the treatment of patients with osteoarthritis knee pain: a 13-week, randomized, placebo-controlled trial. Pain. 2009;146(3):253-60.

9. Koh IJ, Kim MS, Sohn S, Song KY, Choi NY, In Y. Duloxetine Reduces Pain and Improves Quality of Recovery Following Total Knee Arthroplasty in Centrally Sensitized Patients: A Prospective, Randomized Controlled Study. J Bone Joint Surg Am. 2019;101(1):64-73.

10. YaDeau JT, Brummett CM, Mayman DJ, Lin Y, Goytizolo EA, Padgett DE, et al. Duloxetine and Subacute Pain after Knee Arthroplasty when Added to a Multimodal Analgesic Regimen: A Randomized, Placebo-controlled, Triple-blinded Trial. Anesthesiology. 2016;125(3):561-72.

11. Xie J, Ma J, Yao H, Yue C, Pei F. Multiple Boluses of Intravenous Tranexamic Acid to Reduce Hidden Blood Loss After Primary Total Knee Arthroplasty Without Tourniquet: A Randomized Clinical Trial. J Arthroplasty. 2016;31(11):2458-64.

12. Ding ZC, Xu B, Liang ZM, Wang HY, Luo ZY, Zhou ZK. Limited Influence of Comorbidities on Length of Stay after Total Hip Arthroplasty: Experience of Enhanced Recovery after Surgery. Orthop Surg. 2020;12(1):153-61.

13. Xu Z, Zhang H, Luo J, Zhou A, Zhang J. Preemptive analgesia by using celecoxib combined with tramadol/APAP alleviates post-operative pain of patients undergoing total knee arthroplasty. Phys Sportsmed. 2017;45(3):316-22.

14. Lei YT, Xu B, Xie XW, Xie JW, Huang Q, Pei FX. The efficacy and safety of two low-dose peri-operative dexamethasone on pain and recovery following total hip arthroplasty: a randomized controlled trial. Int Orthop. 2018;42(3):499-505.

15. Myles PS, Urquhart $\mathrm{N}$. The linearity of the visual analogue scale in patients with severe acute pain. Anaesth Intensive Care. 2005;33(1):54-8. 
16. Cleeland CS, Ryan KM. Pain assessment: global use of the Brief Pain Inventory. Ann Acad Med Singap. 1994;23(2):129-38.

17. Wong K, Phelan R, Kalso E, Galvin I, Goldstein D, Raja S, et al. Antidepressant drugs for prevention of acute and chronic postsurgical pain: early evidence and recommended future directions. Anesthesiology. 2014;121(3):591-608.

18. Blakely RD, De Felice LJ, Hartzell HC. Molecular physiology of norepinephrine and serotonin transporters. J Exp Biol. 1994;196:263-81.

19. Nutt DJ, Forshall S, Bell C, Rich A, Sandford J, Nash J, et al. Mechanisms of action of selective serotonin reuptake inhibitors in the treatment of psychiatric disorders. Eur Neuropsychopharmacol. 1999;9 Suppl 3:S81-6.

20. Kupers R, Frokjaer VG, Naert A, Christensen R, Budtz-Joergensen E, Kehlet H, et al. A PET [18F]altanserin study of $5-\mathrm{HT} 2 \mathrm{~A}$ receptor binding in the human brain and responses to painful heat stimulation. Neuroimage. 2009;44(3):1001-7.

21. Kupers R, Frokjaer VG, Erritzoe D, Naert A, Budtz-Joergensen E, Nielsen FA, et al. Serotonin transporter binding in the hypothalamus correlates negatively with tonic heat pain ratings in healthy subjects: a [11C]DASB PET study. Neuroimage. 2011;54(2):1336-43.

22. Godlewska BR, Norbury R, Selvaraj S, Cowen PJ, Harmer CJ. Short-term SSRI treatment normalises amygdala hyperactivity in depressed patients. Psychol Med. 2012;42(12):2609-17.

23. Baldwin DS, Stein DJ, Dolberg OT, Bandelow B. How long should a trial of escitalopram treatment be in patients with major depressive disorder, generalised anxiety disorder or social anxiety disorder? An exploration of the randomised controlled trial database. Hum Psychopharmacol. 2009;24(4):269-75.

24. Inase M, Nakahama H, Otsuki T, Fang JZ. Analgesic effects of serotonin microinjection into nucleus raphe magnus and nucleus raphe dorsalis evaluated by the monosodium urate (MSU) tonic pain model in the rat. Brain Res. 1987;426(2):205-11.

25. Mohrland JS, Gebhart GF. Effect of selective destruction of serotonergic neurons in nucleus raphe magnus on morphine-induced antinociception. Life Sci. 1980;27(25-26):2627-32.

26. Proudfit HK. Pharmacologic evidence for the modulation of nociception by noradrenergic neurons. Prog Brain Res. 1988;77:357-70.

27. Pang IH, Vasko MR. Effect of depletion of spinal cord norepinephrine on morphine-induced antinociception. Brain Res. 1986;371(1):171-6.

28. Ho KY, Tay W, Yeo MC, Liu H, Yeo SJ, Chia SL, et al. Duloxetine reduces morphine requirements after knee replacement surgery. $\mathrm{Br} \mathrm{J}$ Anaesth. 2010;105(3):371-6.

29. Blågestad T, Pallesen S, Lunde LH, Sivertsen B, Nordhus IH, Grønli J. Sleep in older chronic pain patients: a comparative polysomnographic study. Clin J Pain. 2012;28(4):277-83.

30. Doghramji K. Sleep extension in sleepy individuals reduces pain sensitivity: new evidence regarding the complex, reciprocal relationship between sleep and pain. Sleep. 2012;35(12):1587-8. 
Figures

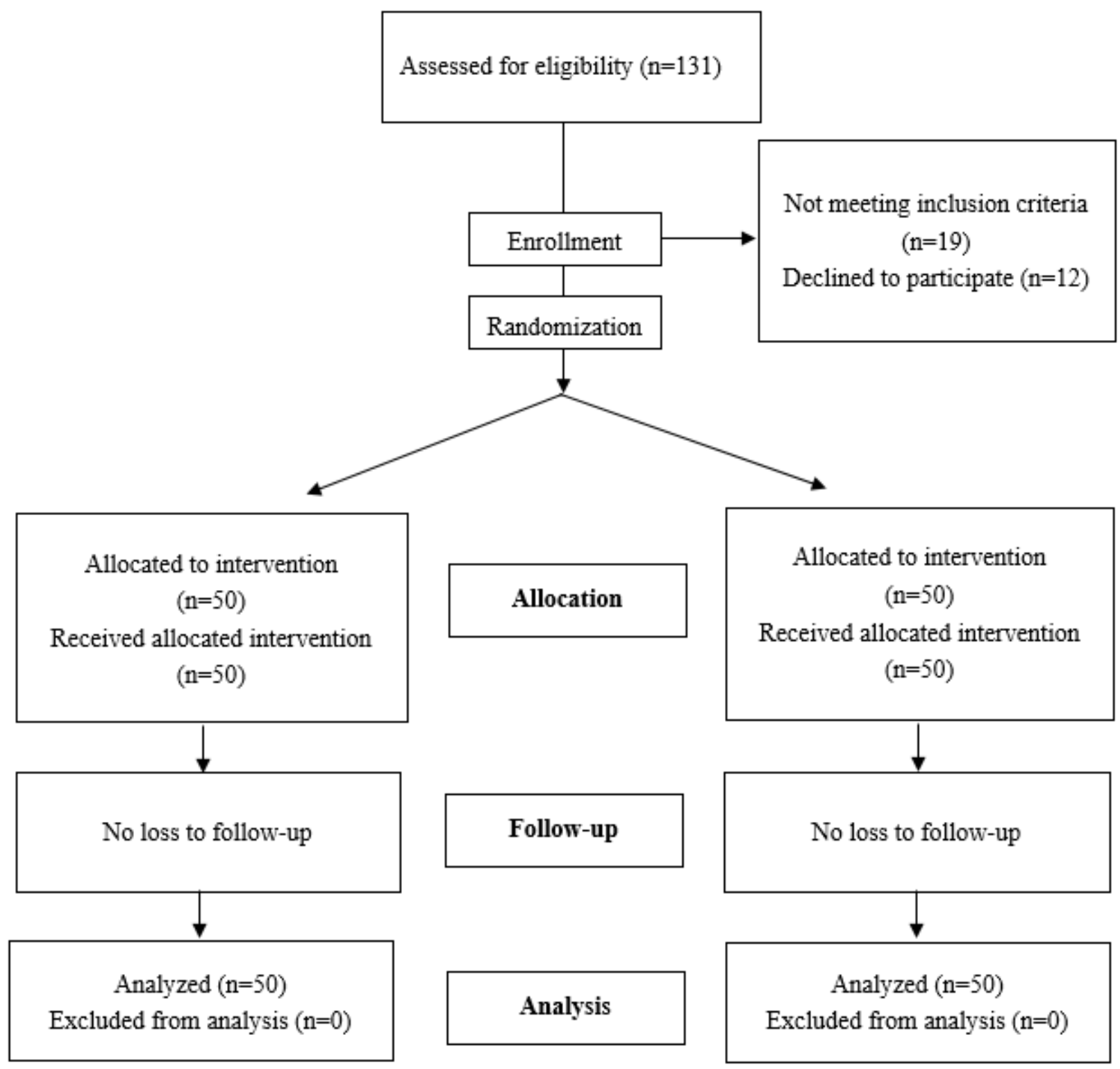

Figure 1

Patients flow chart 


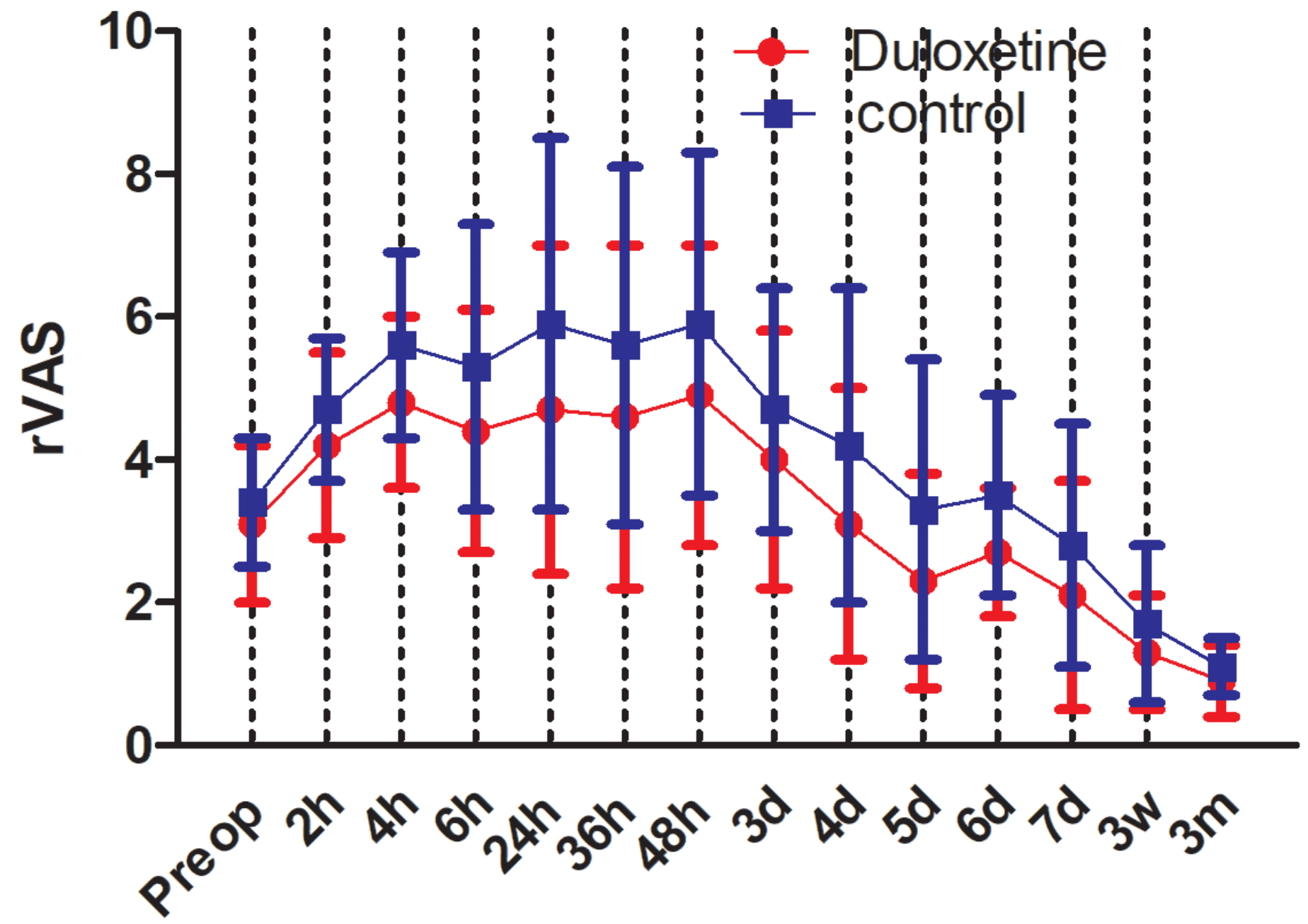

Time

Figure 2

rVAS during the whole study 


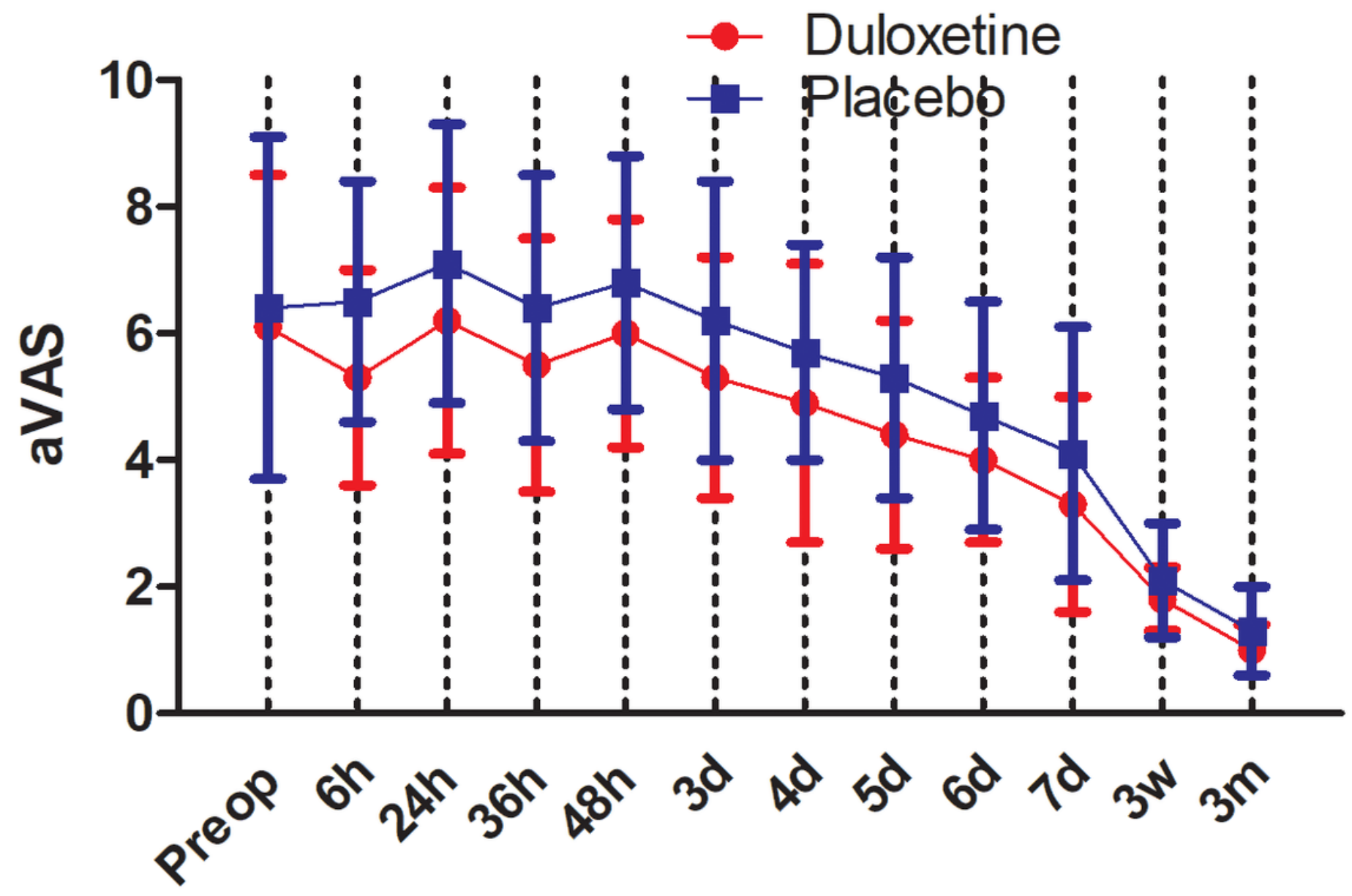

Time

Figure 3

aVAS during the whole study 


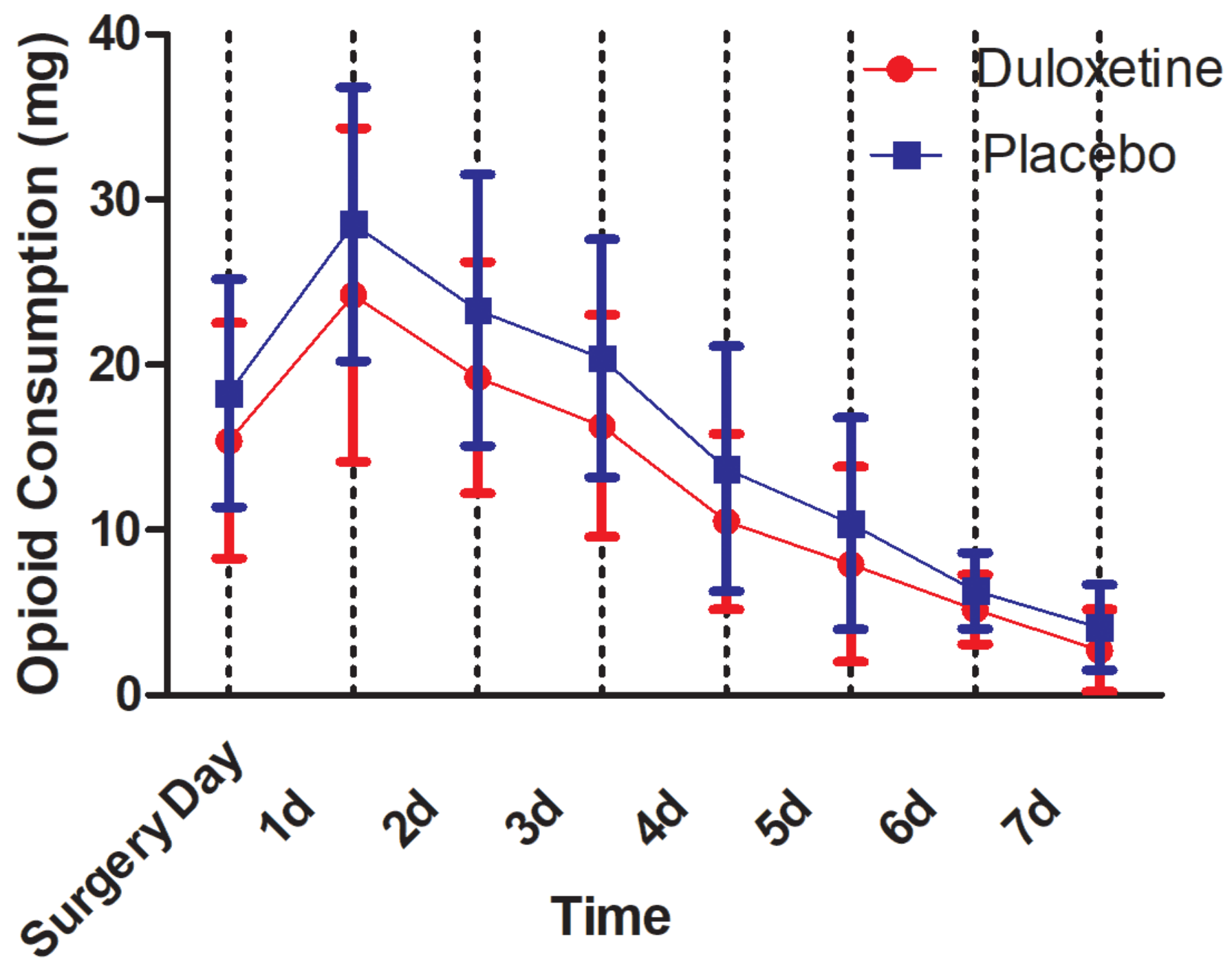

Figure 4

Postoperative opioid consumption 


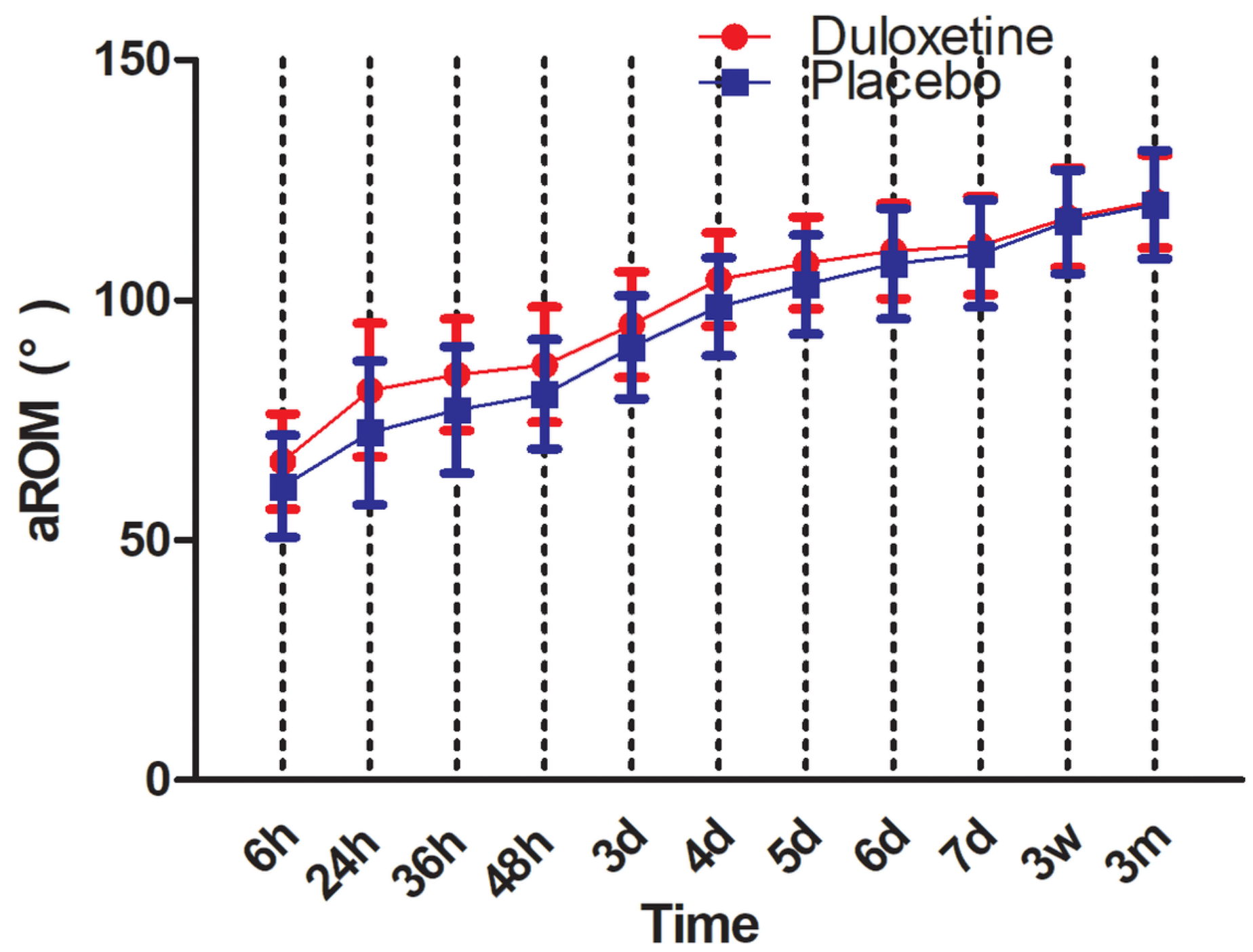

Figure 5

aROM during the whole study 
Data 5

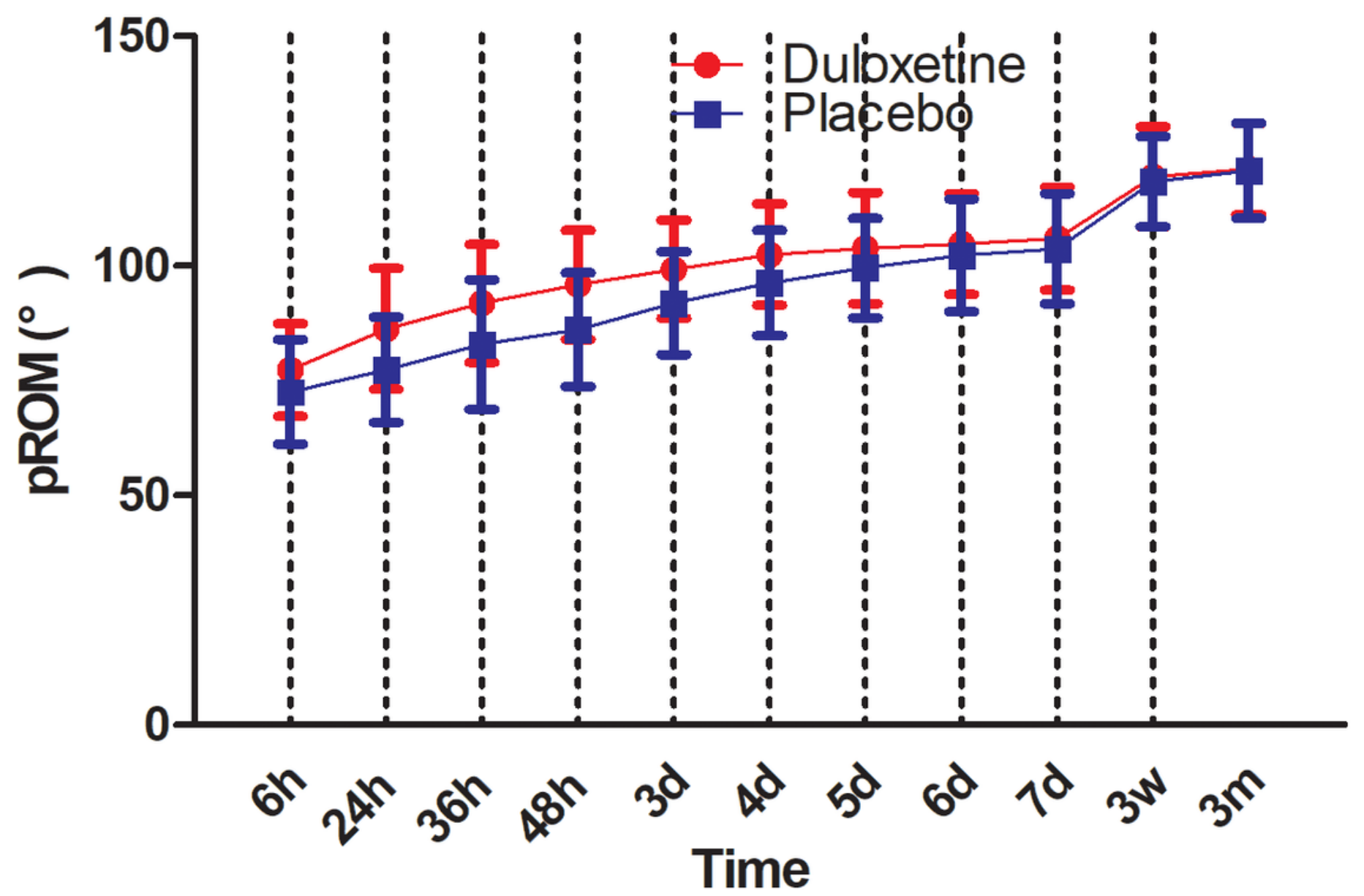

Figure 6

pROM during the whole study 